\title{
Clasificación de gastos por función o por naturaleza para una pyme del sector hotelero en Villavicencio: Estudio de caso
}

\section{Classification of expenses by function or by nature for an SME in the hotel sector in Villavicencio: Case study}

Camila A. Hastamorir Leguízamo

Jorge A. Obando Bastidas ${ }^{2}$

\section{Artículo de investigación}

Recepción: 8/31/2019

Aceptación: 12/15/2019

El Conuco es una revista de acceso abierto revisada por pares. (C) $2018 \mathrm{El}$ autor (es). Este es un artículo de acceso abierto distribuido bajo los términos de la Licencia Internacional Creative Commons Attribution 4.0 (CC-BY 4.0), que permite el uso, distribución y reproducción sin restricciones en cualquier medio, siempre que se acredite el autor y la fuente originales.

Consulte http://creativecommons.org/licenses/by/4.0/. ○OPEN ACCESS

(c) $(\$)$

\section{${ }_{\mathrm{BY}} \mathrm{NC} \mathrm{ND}^{-}$}

1 Contador Público, Universidad de los Llanos, Villavicencio, Colombia.Email.camilaandrea3@gmail.com

2 Master en estadística aplicada, Doctor en formación (Docencia e investigación). Docente investigador Universidad Cooperativa de Colombia Villavicencio, Colombia. Email jorge.obandob@campusucc.edu.co, ORCID: https://orcid. org/0000-0002-4283-2871

Como citar este artículo / To reference this articcle:
ción de gastos por función o por naturaleza para una (investigación, economía y sociedad), 3(1), pp. 1-15.

DOI: https://doi.org/10.22579/2619-614X.528

\section{Resumen}

La clasificación de los gastos acorde a su función o naturaleza cobra importancia en el marco de la convergencia hacia las normas de contabilidad e información financiera en Colombia, en tanto el propósito de la misma es proporcionar información realmente útil para el desarrollo de la industria local. Por tal razón, la presente investigación tuvo como objetivo analizar la clasificación de los gastos como una partida relevante dentro del estado de resultados para una empresa del sector hotelero de la ciudad de Villavicencio. Para tal fin, se utilizó una metodología cualitativa que expone un estudio de caso del sector hotelero de la ciudad de Villavicencio, que permitió identificar y analizar la pyme seleccionada en relación a sus procesos administrativos, contables y de información financiera. Los resultados indican que al tratarse de un área nueva en el campo del conocimiento contable, existe una notable falta de experiencia de los profesionales de la materia, esto ha ocasionado el surgimiento de diferentes interpretaciones y la aplicación de diversos modelos en el desarrollo de la implementación de la nueva normatividad.

Palabras clave. Convergencia, estado de resultados, normas internacionales, pequeñas y medianas empresas.

Códigos JEL: H32, H76, L83 


\section{Abstract}

The classification of expenses depending on their function or nature is very important for compliance with the accounting and financial reporting standards in Colombia, this classification serves the purpose of providing crucial information for the development of the local industry. For that reason, this research aimed to analyze the categorization of expenses as a relevant item within the balance statement for a hotel company in the city of Villavicencio. For this purpose, a qualitative methodology was used to present a case study of the hotel business of the city of Villavicencio, which allowed the identification and analysis of the selected Small and Medium-sized Enterprise (SME) in relation to its administrative, accounting and financial information processes. The results indicate that since this is a new area in the field of accounting knowledge, there is a notable lack of experience from the professionals in this field, which has led to the emergence of different interpretations and the implementation of various models while applying the new accounting regulations.

Keywords. Convergence, income statement, international financial reporting standard, small and medium-sized businesses.

\section{Introducción}

El Consejo Técnico de la Contaduría Pública (CTCP) y la Superintendencia de Sociedades han realizado esfuerzos para facilitar herramientas de carácter general que orienten y apoyen a los empresarios en el proceso de convergencia a Normas Internacionales de Información Financiera (NIIF). De esta misma forma, para que dichas herramientas sean una guía para los profesionales de la Contaduría Pública que den luz sobre cómo iniciar este proceso en las diferentes esferas del universo empresarial.

Desde una mirada al mundo respecto a los sistemas contables y de información financiera Cano (2010), expresa que:

La globalización de la economía y de la información, su internacionalización y la expansión económica que se está viviendo ahora en la gran mayoría de países, han llevado a que las empresas empiecen a replantear su futuro, al enfocar su participación en la economía global. (p.125)

Bajo esta premisa, Colombia ha decidido ingresar en esta dinámica con el proceso de convergencia hacia las normas de contabilidad e información financiera y de aseguramiento de la información, con estándares internacionales, desatando toda una revolución, no sólo en la industria sino en el que hacer de la profesión contable.

De esta manera en Colombia, dicho proceso inició con la expedición de un marco normativo bajo la Ley 1314 en el año 2009 y posterior el Decreto Reglamentario 3022 emitido en el año 2013, el cual define y clasifica las entidades pertenecientes al grupo 2 y las normas que le aplican, para el caso, NIIF para pequeñas y medianas empresas (pymes) acogido al modelo del Consejo de Normas Internacionales de Contabilidad, por sus siglas en inglés International Accounting Standards Board (IASB).

Se puede señalar que las razones que condujeron a la búsqueda de una adopción de normas en materia de contabilidad, revelación de información financiera y aseguramiento de la información bajo estándares internacionales de aceptación mundial, se sustentan en la necesidad de apoyar en un contexto económico la dinámica comercial colombiana, a través de instrumentos que le permitan acceder a mercados internacionales, captar 
la inversión extranjera y aportar así al desarrollo económico del país (Consejo Técnico de la Contaduría Pública, 2012).

Al respecto, Mejía, Montes \& Montilla (2008) afirman sobre el modelo IASB que:

Si se unifica el proceso contable a partir de la propuesta IASB, se desconocería la multiplicidad de usuarios de la información contable y la variada gama de necesidades y de entornos existentes. Esta propuesta responde a unos usuarios en particular, a unas necesidades específicas y a un desarrollo empresarial concreto. Estas condiciones no son precisamente similares a las de los países subdesarrollados. (p. 64)

Aunque en otro sentido, Díaz, Gil \& Vílchez (2010), afirman que este marco conceptual es aplicable a una amplia gama de modelos contables, suministrando una guía al preparar y presentar los estados financieros por medio del modelo escogido.

De cualquier manera, todo cambio trae consigo diferentes obstáculos, Hellmann, Perera \& Patel (2010), presentan diversos problemas que se generaron con la adopción de las NIIF en Alemania, como: problemas de traducción de las NIIF, distintas interpretaciones de las NIIF, falta de personal cualificado, entre otros. De allí, la importancia de orientar ayudas hacia segmentos económicos específicos.

Todo lo anterior, trasciende en la relevancia que tienen los estados financieros para una entidad, pues estos representan el producto final del proceso contable, cuyo propósito principal es "informar a los distintos usuarios, sobre el resultado de las operaciones llevadas a cabo en la entidad, durante un periodo determinado, su situación financiera y sus flujos de efectivo, proporcionando información que sea útil para la toma de decisiones" (Marcotrigiano, 2013, p. 46).
A continuación, entre el grupo de estados financieros postulados por la IASB, se encuentra el estado del resultado integral, definido por Marcotrigiano (2013) como "el estado financiero que muestra el resultado de las operaciones llevadas a cabo por la entidad y la rentabilidad de sus operaciones" (p. 60), cuya importancia radica en la determinación del rendimiento y sostenibilidad a futuro de la empresa. Dentro de su estructura de ingresos y gastos, la norma ofrece a bien la posibilidad de clasificar los gastos, dada su naturaleza o función, seleccionando aquel que mejor presente los elementos del desempeño de la empresa.

En este sentido, la presente investigación tuvo como objetivo analizar la clasificación de los gastos como una partida relevante dentro del estado del resultado integral de una empresa del sector hotelero de la ciudad de Villavicencio, Meta, Colombia, soportada en el aporte de Humphrey \& Scapens (1992, citado en Salazar, 2013) quienes se refieren a la importancia de la elaboración de casos de estudios, en especial en las ciencias contables, dado que de allí se prueban hipótesis y teorías a escala mayor, o partir de ello se pueden desarrollar implicaciones teóricas sobre las prácticas contables.

La investigación se aborda desde una metodología cualitativa que busca conocer el proceso de convergencia hacia la NIIF de una empresa del sector hotelero de la ciudad de Villavicencio catalogada como pyme, a través de un estudio de caso. Para tal fin, se utilizaron múltiples fuentes de datos bajo el principio de la triangulación recomendada por Yin (1993, citado en Martínez, 2006), el cual busca confrontar la información obtenida a través de distintas fuentes con el fin garantizar la validez interna de la investigación, lo que permitió verificar los datos obtenidos a través de distintos instrumentos de recolección 
de información, tales como: la ficha de caracterización, la encuesta y la lista de chequeo.

Para ello, el proceso de obtención de la información se dividió en dos fases; la primera consistió en categorizar y caracterizar la empresa por medio de la encuesta y la ficha de caracterización, realizadas a dos agentes privilegiados con el propósito de conocer sobre aspectos contables y financieros. En la segunda fase, se aplicaron, analizaron y discutieron listas de chequeo por cada una de las secciones contenidas en el Decreto 3022 de 2013 aplicables a las pymes, basadas en los conceptos de reconocimiento y medición, con el fin de conocer la orientación de sus gastos, y ahondar sobre su proceso de convergencia hacia la norma internacional.

\section{Normas Internacionales de Información Financiera como estrategia de armonización contable mundial}

Las NIIF son desarrolladas y aprobadas por la IASB (International Accounting Standards Board), una organización independiente del sector privado sin ánimo de lucro, cuyos objetivos son promover el uso y la aplicación de las normas, así como llevar a la convergencia entre las normas contables nacionales y las normas internacionales de contabilidad y de información financiera, hacia soluciones de alta calidad. Las NIIF hacen referencia a un conjunto de normas contables legalmente exigibles, globalmente aceptadas, comprensibles y de alta calidad, basadas en conceptos básicos y reglas que deben ser observadas al registrar la información financiera (Bohórquez, 2015).

Antes de la creación de la ISAB en el año 2001, el organismo encargado de emitir las Normas Internacionales de Contabilidad (NIC) era el IASC (International Accounting Standards Committee), organismo que nació en 1973 a través del acuerdo de representantes de profesiones contables de varios países, que buscaban una serie de normas que pudieran ser aplicadas a nivel general, con el fin de favorecer la armonización, la información y su comparabilidad (Nicniif. org, 2016; Bohórquez, 2015). Así mismo, escándalos de carácter económico que estallaron alrededor del mundo suscitaron transformaciones en materia de regulación contable.

Actualmente, la Unión Europea conformada por 27 estados y una centena más de países aplican NIIF. En América, los Estados Unidos, América Central, Bolivia, Canadá, Ecuador, Guyana, Perú, Uruguay, Venezuela, Argentina, Brasil y Chile ya han concluido el proceso de adopción. Colombia aún se encuentra en dicho proceso; pese a que el Decreto 2649 emitido en el año 1993 por el presidente de la República de Colombia, que regulaba el tratamiento contable, fue una adaptación de las Normas Internacionales de Contabilidad de la época, las realidades económicas del mundo son otras (López y Zea, 2010, p.p. 103, 104).

\section{Adopción de las NIIF en Colombia}

Mediante la promulgación de la Ley 1314, emitida en el año 2009 por el congreso de Colombia, se dispuso la modernización de las normas contables para mejorar la productividad, la competitividad y el desarrollo armónico de la actividad empresarial por medio de la convergencia hacia estándares internacionales reconocidos, facultando al CTCP como la autoridad colombiana de normalización técnica de las normas contables de información financiera y de aseguramiento de la información, por ello es de su competencia elaborar las propuestas que son la base a partir de la cual los ministerios de Hacienda y Crédito Público, y de Comercio, Industria y Turismo expiden de manera conjunta, principios, normas, 
interpretaciones y guías de contabilidad e información financiera y de aseguramiento de la información (CTCP, 2012).

Por consiguiente, para dar cumplimiento a lo dispuesto en la Ley 1314, el Consejo Técnico de la Contaduría Pública emitió el documento del Direccionamiento Estratégico en el año 2012, que tuvo como objetivo: "orientar el desarrollo efectivo del proceso de convergencia hacia las normas de contabilidad e información financiera y de aseguramiento de la información, con estándares internacionales" (CTCP, 2012, p.1); este documento sugiere la aplicación de las normas de contabilidad e información financiera de manera diferencial por tres grupos de usuarios, grupo 1: NIIF plenas; grupo 2: NIIF para pymes; grupo 3: contabilidad simplificada.

Por lo que se refiere al grupo 2, trata de las pequeñas y medianas empresas; en Colombia según la Ley 590 del 2000 y posterior modificación por la Ley 905 del 2004, emitidas por el congreso de Colombia, se determinó la clasificación de estas empresas, teniendo en cuenta que cumplieran las siguientes características: activos totales desde quinientos uno (501) hasta treinta mil (30.000) Salarios Mínimos Mensuales Legales Vigentes (SMMLV) o planta de personal entre once (11) y doscientos (200) trabajadores, que no sean emisores de valores ni entidades de interés público. Otra condición para este grupo la establece la sección 1 del Decreto 3022 de 2013, cuando se refiere a que "no tienen obligación pública de rendir cuentas y publican estados financieros con propósito de información general".

\section{NIIF para pymes}

La economía en el mundo se sustenta en las pequeñas y medianas empresas. "En Europa se estima que hay más de 7000 entidades de interés público (grandes empresas) y más de un millón de entidades privadas (negocios pequeños y medianos)" (Wong, 2004, citado en Espinosa, 2015, p. 19). Las pymes representan una de las principales fuerzas en el desarrollo económico, especialmente en los países en vías de desarrollo; particularmente en Colombia las pymes representan el 94\% de la economía de los negocios, constituyen el 95\% del empleo en el comercio y el $74 \%$ de la producción (Mejía, 2005).

En cuanto a la NIIF para pymes, esta fue diseñada para satisfacer las necesidades y capacidades de las pequeñas y medianas empresas. En comparación con las NIIF completas, la NIIF para las pymes es menos compleja en una serie de formas para tratar ciertos eventos y transacciones en los estados financieros (Nicniif.org, 2016). Otra es la opinión que le merece a los autores Mejía, Montes \& Montilla (2008), quienes afirman que "en sus capítulos, muchos de ellos están destinados a prescribir los tratamientos contables propios de entidades grandes, por lo que no corresponden propiamente a las condiciones típicas de las pymes" (p. 79).

En tal sentido, el Grupo de Trabajo Intergubernamental de Expertos en Normas Internacionales de Contabilidad y Presentación de Informes (Intergovernmental Working Group of Experts on Internacional Standards of Accouting and Reporting, ISAR), reconoció en el año 2002 que la mayoría de sus estándares contables habían sido elaborados para las grandes empresas, y que por tanto, las pequeñas y medianas entidades han experimentado diferentes dificultades para cumplirlos, acordando trabajar en la identificación de estos problemas para la búsqueda de soluciones.

Por lo que se refiere al proceso de convergencia en Colombia, el CTCP, el 22 de diciembre de 2010, realizó una solicitud abierta a la comunidad 
en general para realizar aportes y comentarios al documento Propuesta para el direccionamiento estratégico del CTCP y el entendimiento común del proceso de convergencia de las normas de contabilidad, de información financiera y de aseguramiento de la información, con estándares internacionales, la cual tuvo un plazo inicial para entregar comentarios hasta el 31 de enero del 2011, donde se presentaron 3 opiniones; seguidamente se amplió el plazo hasta el 28 de febrero, concluida esta prórroga se recibieron 48 participaciones (López \& Zea, 2011).

Lo anterior, demuestra que la convocatoria realizada por el CTCP, dada la exigua participación de grupos de interés, preparadores y aseguradores de la información, no surtió el efecto esperado. Al respecto, Mejía, Montes \& Montilla (2008), afirman que la trascendencia del cambio en el núcleo teórico que fundamenta la normativa contable, debe resultar de un amplio debate, donde confluyan los actores sociales, políticos y económicos afectados por su implementación; lo que sugiere que la normatividad implantada desconoce los efectos económicos, sociales y culturales que trae consigo.

\section{La profesión contable como eje fundamental en la convergencia}

La disciplina contable, en el contexto colombiano, es protagonista de las transformaciones que en materia contable y financiera han sobrevenido con la nueva regulación gracias al proceso de convergencia a las NIIF. Desde el punto de vista académico, Ferrer (2013), se refiere a las facultades de contaduría pública de las universidades como las responsables del cambio en la visión de la contabilidad hacia una perspectiva internacional, en aras de suplir la necesidad de actualización de los contadores públicos.

Por su parte, autores como Mejía, Montes \& Montilla (2008), se refieren a la aceptación de los estándares internacionales de la profesión contable como una entrega de la autonomía en materia de regulación nacional, que endosa la potestad de las naciones de autorregularse y entrega a los organismos internacionales la autoridad de normalizar el ejercicio profesional; recomendando así, concentrar los esfuerzos académicos en el fortalecimiento de la educación y la formación en epistemología, teoría contable, historia contable universal e historia del desarrollo de la profesión contable y la regulación del país.

Al mismo tiempo, Ospina \& Villaquirán (2010), afirman que en Colombia el producto de la contabilidad no hace referencia a los aspectos del análisis gerencial, estratégico y político de la empresa, situación que es atribuida al contador como una desconexión con el entendimiento del negocio, como una extralimitación de su función hasta el acto de entrega de información al gerente. Ferrer (2013), asegura que la producción del contador es útil para cumplir con obligaciones tributarias y requerimientos de carácter estatal, hecho que no responde al cometido de su profesión.

Desde otra perspectiva, se habla de la postura crítica y constructiva que debe ejercer el contador público en la sociedad; Guarín (2003, citado en Mejía, Montes \& Montilla, 2008), se refiere a una sumisión del experto, especialista, que hace parte de la maquinaria burocrática, justificando su neutralidad en el quehacer científico, con restricción a la libertad de pensamiento y sacrificio de su creatividad e ingenio, entregando su técnica profesional al dominio de las más altas atmósferas; Gil (2004, citado en Mejía et al., 2008), expone la contabilidad como un conocimiento que no se utiliza para cuestionar sino para validar.

Esta y otras apreciaciones dejan en cuestión el rol de la profesión contable y su experticia en los estándares internacionales de contaduría para 
atender el desafío que representa no sólo su aplicación, sino la formación de otros profesionales en la materia. En este sentido, el ahínco del profesional en contaduría pública debe orientarse a la responsabilidad para preservar la equidad entre distintos grupos, en busca del uso apropiado de sus habilidades y capacidades.

\section{Los estados financieros}

Los estados financieros representan el producto final del proceso contable. El Decreto 3022 de 2013, asigna a los estados financieros dos objetivos básicos: el primero de ellos es proporcionar información sobre la situación financiera, el rendimiento y los flujos de efectivo de la entidad que sea útil para la toma de decisiones económicas en una amplia gama de usuarios que no están en condiciones de exigir informes a la medida de sus necesidades específicas de información; y el segundo, mostrar los resultados de la administración llevada a cabo por la gerencia, que dan cuenta de la responsabilidad en la gestión de recursos confiados a la misma.

Por su parte, Díaz et al. (2010) se refieren a la relevancia de la información contenida en los estados financieros, dado que esta influye en las decisiones económicas de los usuarios. Respecto al nuevo marco normativo, Mejía et al. (2008) mencionan que los estados financieros de propósito general desconocen la importancia de propietarios y administradores como usuarios de la información, orientándose únicamente a usuarios externos, dado el contexto de la normativa que desarrolló la IASB, el cual buscó conservar la uniformidad y espíritu de los estándares del modelo bursátil.

Fundamentalmente, un conjunto completo de estados financieros se compone del estado de situación financiera, estado del resultado integral, estado de cambios en el patrimonio y estado de flujos de efectivo, que se constituyen de cinco elementos básicos: activo, pasivo, patrimonio, ingresos y gastos. Dichos estados son tratados por la NIIF para pymes en las secciones 3, 4, 5, 6, 7 y 8 del Decreto 3022 de 2013, allí se hace mención de sus aspectos generales, tratamiento específico y notas; también se enuncia las características cualitativas que deben cumplir para que la información sea útil.

Específicamente, las características cualitativas de los estados financieros son comprensibilidad, relevancia, materialidad o importancia relativa, fiabilidad, esencia sobre la forma, prudencia, integridad, comparabilidad, oportunidad, y equilibrio entre costo y beneficio. En ellas se soporta el deber ser del contador público, no sólo respecto a sus conocimientos y habilidades para desarrollar informes financieros que proporcionen información valiosa a la organización, sino en el ejercicio de la conciencia ética y la conducta moral en su actividad profesional para lograr gestiones impecables (Montaner \& Perozo, 2008).

Ahora bien, dando continuidad al estado financiero objetivo, la Fundación IFRS (2010) define el estado del resultado integral como el estado financiero "con propósito de información general (...) que proporciona información sobre la situación financiera, el rendimiento y los flujos de efectivo de la entidad" (p. 2). En él deben reflejarse todas las partidas de ingreso y gasto reconocidas en el periodo (Decreto 3022 del 2013), cuya relación determinará el rendimiento y razón de ser de la empresa: la obtención de lucro como compensación a la inversión de los propietarios.

\section{Clasificación de los gastos en una pyme del sector hotelero}

Como paso previo al estudio de la clasificación de los gastos dentro del estado del resultado in- 
tegral, resulta importante definir el gasto dentro del contexto de la contabilidad. La definición de gasto incluye tanto las pérdidas como los gastos que surgen en las actividades ordinarias de la entidad. Los gastos que surgen de la actividad ordinaria incluyen el costo de las ventas, los gastos de personal, los honorarios, la depreciación, entre otros. Las pérdidas también cumplen la definición de gastos pero no surgen en el curso de las actividades ordinarias de la entidad (Salazar, Salazar \& Marín, 2015).

También es válido aclarar que, el costo es el que tiene relación directa con el producto o servicio vendido, y el gasto es el que no teniendo relación directa con la venta, está ligado al periodo de tiempo que se analiza en el estado del resultado integral (Menéndez, 2010). En este sentido, el Decreto 3022 de 2013 deja abierta la posibilidad de un desglose de gastos dentro del estado del resultado integral, a través de dos métodos: desglose por naturaleza de los gastos y desglose por función de los gastos.

Ahora bien, el desglose por naturaleza consiste en la agrupación de los gastos de acuerdo a su tipo, como puede ser: salarios y otros beneficios a empleados, depreciaciones y amortizaciones, deterioro del valor de los activos, dotación de provisiones, entre otros. El desglose por función consiste en la agrupación de los gastos de acuerdo con su uso o destino, como puede ser: costo de ventas, gastos de actividades de ventas, gastos de administración, o cualquier otra categoría representativa para la entidad (Salazar et al., 2015).

De esta manera, la selección de uno u otro método se condiciona al objetivo de proporcionar información fiable y relevante dentro de la entidad, para los usuarios de dicha información. A continuación se estudian diferentes posturas:
- Alexander \& Archer (2005), afirman que ambos enfoques del desglose de gastos, tienen sus ventajas y sus desventajas. Sin embargo, aseguran que el desglose de los gastos atendiendo a su naturaleza, requiere preparar menos análisis, pero "es discutiblemente menos informativo y tiene la desventaja lógica que quizás parezca implicar que los cambios en el inventario son un gasto (o un ingreso) operacional" (p. 319).

- Sánchez \& Holguín (2007), sugieren que debe considerarse el modelo de presentación por naturaleza, atendiendo esta sugerencia como una orientación para una empresa del sector servicios, y no a la producción industrial. Sustentan su argumento en el grado de complejidad que implica el hecho de realizar una clasificación de gastos por función, puesto que no siempre es posible determinar con exactitud la proporción a asignar de un gasto, a cierta área o departamento.

- Menéndez (2010), realiza una investigación basada en la optimización de costos en la gestión hotelera, orientada en un análisis de cada uno de los departamentos que intervienen en el funcionamiento de un hotel. Indica que, la implementación de toda una estrategia de optimización de costos, requiere de un adecuado sistema de control de gestión, de forma que pueda analizarse al detalle la situación actual e identificar las posibles áreas de mejora; para ello es imprescindible el desglose por función de los gastos.

- Martínez (2009), a través de su investigación sobre la evaluación financiera y operacional en pymes del orden hotelero en Colombia, se refiere a la estructuración de los resultados operacionales y los criterios de localización de costos, a través de la concentración de costos y gastos en: costo de ventas, costos de personal 
y otros gastos. Lo anterior permite inferir que el método de desglose de gastos empleado es de acuerdo a su naturaleza.

- Ascanio (2005), menciona las ventas departamentales, los costos y gastos operativos y generales, dentro de un universo de indicadores a estudiar para determinar la viabilidad de un nuevo proyecto hotelero. Considera que el estudio de los ingresos y gastos a través de departamentos, puede hacer o no plausible la posibilidad del establecimiento de un hotel, proyectando su punto de equilibrio y rentabilidad. Esto es, clasificación de gastos acorde a su función.

\section{Método}

Como desarrollo de la primera fase de la obtención de la información, utilizando el principio de triangulación de Yin (1993, citado en Martínez, 2006), se empleó una ficha de caracterización y una encuesta con preguntas de orden general aplicadas al gerente y contador de la empresa, entrevistas directas, observación directa, observación de los participantes e instalaciones u objetos físicos (Chetty, 1996, citado en Martínez, 2006). Con estos instrumentos se obtuvo información como datos generales de la sociedad, aspectos socioeconómicos, administrativos, contables y financieros.

\section{Categorización y caracterización}

Así, dadas sus características, y de acuerdo a la Ley 1314 de 2009 y el Decreto Reglamentario 3022 de 2013, el cual define y clasifica los miembros del grupo 2 (pymes), se concluye que el hotel contexto de estudio, se ajusta a los requerimientos para la aplicación de NIIF para pymes: Los montos de sus activos e ingresos no superan los 30.000 SMMLV, su planta de personal no supera los 60 empleados, no es una empresa emisora de valores, no tiene sucursales en el extranjero, tampoco es sucursal ni subordinada, es una empresa que no maneja ni recauda ni emite fondos del público, no presta servicios públicos ni está vigilada por la Superintendencia Financiera de Colombia.

En particular, dando cuenta de sus características, la planta promedio de personal es de 56 empleados vinculados de manera directa; debido a la estacionalidad de sus servicios, en temporada alta puede llegar a vincular adicionalmente a $30 \mathrm{em}$ pleados en el área operativa, a través de terceros; la cantidad de proveedores que surten al hotel oscilan entre 11 y 30, con la mayoría de ellos no maneja ningún tipo de financiación, pues se trata de alimentos perecederos y similares. Su volumen de clientes mensuales es superior a 50, en su mayoría con pagos al contado de los servicios prestados; las facturas a crédito son expedidas con un plazo no mayor a 30 días, la cartera actual es en su totalidad de los socios.

Además, cuenta con un mecanismo de reservas mediante portales de internet de orden internacional, estas operaciones se realizan en moneda extranjera, y tiene un costo que oscila entre el 12 y $22 \%$ sobre el valor total del servicio; dicha comisión es causada con la tasa de cambio representativa del mercado (TRM) del momento de la reserva, generando una diferencia en cambio al momento del pago si la TRM ha variado. Su software contable ' $\mathrm{J} 3$ ' no se encuentra parametrizado bajo la norma internacional, y no representa gran diferencia respecto al software contable de empresas dedicadas a otras actividades económicas. Su gerente ha sido reemplazado en tres ocasiones los dos últimos años, lo que denota una debilidad en su órgano administrador.

En su aspecto contable y financiero, la entidad cuenta con un contador público de tiempo completo, quien es el director financiero de la misma, 
y un equipo de trabajo compuesto por un auxiliar contable, una coordinadora de centros de costos y dos auditores (diurno y nocturno), quienes son los encargados de verificar que todos los cargos de habitación de la jornada (comandas) estén bien soportados de acuerdo al cargo a la cuenta, descontando el depósito previo al momento de la reserva, así mismo, de verificar que todo los pagos correspondan a los consumos del cliente.

Por otra parte, lo que concierne a sus obligaciones tributarias, la empresa debe presentar información exógena, declaración del Impuesto al Valor Agregado (IVA), declaración de retención en la fuente a título de renta, autorretención del Impuesto para la Equidad (CREE), impuesto a la riqueza, impuesto al consumo, retención del impuesto de Industria y Comercio (ICA), impuesto al turismo. Adicionalmente, está en la obligación de suministrar información a la Superintendencia de Sociedades, Cámara de Comercio, y otras entidades que se encargan de manera específica de vigilar su actividad de prestación de servicios de alojamiento, como es la Asociación Hotelera de Colombia (COTELCO), Departamento Administrativo Nacional de Estadística (DANE), y Secretaria de Turismo Departamental y Municipal.

Respecto al proceso de preparación y aplicación del nuevo marco normativo, cuyo periodo de preparación obligatorio fue establecido entre el 01 de enero de 2014 y el 31 de diciembre de 2014, el periodo de transición entre el 01 de enero de 2015 y el 31 de diciembre de 2015, y el periodo de aplicación obligatoria a partir del 01 de enero de 2016, sólo está en conocimiento del representante legal y el departamento financiero, no se tiene aún previsto actualizaciones tecnológicas requeridas por la transición de norma, no existe un cronograma de aplicación dentro de la entidad, ni un presupuesto estimado para ello; si bien se han realizado discusiones respecto al proceso de convergencia a NIIF, la organización en general no se encuentra en proceso de preparación.

Por último, en desarrollo de la segunda fase de obtención de la información, en la que fue aplicada la lista de chequeo por cada una de las secciones contenidas en el Decreto 3022 de 2013 aplicables a las pymes, basada en los conceptos de reconocimiento y medición, orientándose específicamente hacia los gastos, se obtuvo como resultado el siguiente análisis:

En el Hotel, existen funcionalmente 8 dependencias que se distribuyen entre restaurante y bar, lavandería, alojamiento, mercadeo y ventas, mantenimiento, contabilidad y finanzas, recursos humanos y administración (área gerencial). Esta es una apreciación tomada a partir de la observación directa, de los participantes e instalaciones, puesto que en el departamento contable así como en el aspecto organizacional dicha división no está definida; a partir de allí fue efectuada la lista de chequeo que consistió básicamente en determinar el origen del gasto en cada una de estas áreas y su destino final, es decir, la forma de su representación dentro del estado del resultado integral.

El restaurante y bar, y lavandería son administrados bajo el criterio de centros de costos y cuentan con un responsable dentro de la administración con el fin de llevar un control más juicioso de sus inventarios y suministros. Dentro de los demás departamentos operacionales, es decir, que son susceptibles de generar un ingreso directo al hotel, no existe un tratamiento similar para el control de sus costos; los gastos de las denominadas divisiones funcionales o no operativas son tratados de manera ordinaria, es decir, periódicamente.

Ahora bien, en el restaurante y bar, bajo el control del centro de costos, se encuentran elementos tales como: licores, bienes fungibles o insumos 
que se consumirán en el proceso productivo, sean condimentos, aceites, sal, azúcar, entre otros, y materias primas como harina, arroz, carnes, verduras, frutas, legumbres, embutidos, congelados; no se contemplan costos de personal, lavandería, suministro de energía, agua, gas, dentro del coste final de producto vendido.

Seguidamente, en el área de lavandería, también bajo el control de centro de costos, se encontró variedad de productos químicos para el tratamiento de lencería (sábanas y toallas), detergentes, suministro de energía, agua y gas; no se contempla gastos de personal ni mantenimiento. Por otra parte, los gastos arrojados por todo lo que comprende el proceso de lavandería no son redistribuidos en las áreas que tienen algún vínculo o requieren de este servicio para su funcionamiento.

El área de alojamiento, comprendida por 115 habitaciones, involucra personal de recepción, personal de limpieza, lavandería de lencería y mantenimiento; bajo estos eventos se observa una participación de diferentes áreas operativas dentro de la prestación del servicio, no obstante, se advierte la ausencia de una concentración de gastos para esta actividad, los cuales son cargados al concepto desde el cual tuvieron origen, respectivamente. Por ejemplo, los costes de lavado de ropa de las habitaciones son cargados a la lavandería, en lugar de ser imputados al departamento de alojamiento.

De la misma manera, la división de mantenimiento se ocupa de una variedad de tareas tales como: mantenimiento de jardines, zona húmeda, máquinas (lavandería, planta de energía, calentadores y piscina), reparaciones locativas, entre otros; los gastos allí generados son reflejados como gastos operativos, independientemente del área donde haya dado lugar la reparación o mantenimiento, por ejemplo, la reparación de un archivador en la oficina del director de recursos humanos.

Por otro lado, uno de los conceptos de mayor relevancia y complejidad en el funcionamiento de una empresa hotelera es el personal, este puede llegar a representar más del 50\% del total de gastos del hotel, puesto que incluye no sólo sueldos, salarios y seguridad social, sino también prestaciones sociales, gastos de alojamiento, manutención, formación del personal, entre otros (Menéndez 2010); en referencia al tratamiento de gastos de la empresa contexto de estudio, los trabajadores vinculados de manera directa, se encuentran clasificados entre el personal de administración y ventas únicamente.

En lo que respecta al área de mercadeo y ventas, como es común, el tratamiento de sus gastos desemboca en los gastos de operación conforme a la presentación tradicional de un estado de resultados; de la misma forma se presentan los gastos de los departamentos no operativos: recursos humanos, departamento contable y financiero, departamento administrativo o gerencial, los cuales son transcritos a los gastos de administración dentro del estado financiero ya mencionado.

De modo accesorio, los gastos por concepto de suministro de energía, agua potable, gas natural, telecomunicaciones, son prorrateados para su presentación como gastos de administración y operación, respectivamente, dentro del estado del resultado integral; las compras de insumos para las diferentes áreas del hotel son autorizadas desde el departamento financiero, donde únicamente son individualizadas las correspondientes al restaurante y bar, y lavandería, como se había mencionado anteriormente; labores como la seguridad del hotel son tercerizadas a través de una compañía de vigilancia y su costo es reflejado como un gasto de operación. 


\section{Conclusiones}

En la empresa estudiada, la presentación del estado del resultado integral empleada por la organización es la representación tradicional del antiguamente llamado estado de resultados bajo los Principios de Contabilidad Generalmente Aceptados (PCGA) en Colombia, en él se muestran los gastos en dos grandes grupos: gastos de administración y gastos de operación; podría decirse que este es un desglose por función pero de una manera muy general si lo que se pretende es informar en detalle los gastos generados por cada una de las áreas del hotel.

Así, la trascendencia del cambio a la normatividad internacional radica en la puntualidad y veracidad de la información contable y financiera, en aras de reflejar una situación económica real de la organización. En consecuencia, la unidad de análisis o contexto de estudio debe resolver situaciones como la definición de los departamentos que la conforman, de forma que le permita realizar una verdadera optimización de costos, y tomar medidas frente a las situaciones que estén afectando sustancialmente su condición financiera.

En consecuencia, en el caso estudiado existen argumentos para señalar que los estados financieros son empleados como una herramienta para legitimar las operaciones ante las entidades de control y ante la sociedad, lejos de reflejar la real situación financiera de la entidad, donde el profesional responsable de los asuntos contables de la empresa desconoce la trascendencia del proceso de convergencia a la normatividad internacional, en tanto, ello implica la disgregación de información y consolidación en nuevos procesos que reflejen con mayor fidelidad la situación financiera de la organización.

Esta investigación, orientada bajo un estudio de caso, cobra relevancia en tanto refleja la situación real de una pyme hotelera en Villavicencio, Meta, Colombia; su desinterés en la adopción de los estándares internacionales como un rasgo cultural marcado en la rama gerencial que se generaliza en las empresas locales, que ejerce gran influencia en una labor de trascendencia en el ámbito organizacional y financiero que, si bien es cierto, atañe a la labor del contador público pero, no lo hace responsable de la oportuna ejecución de la misma.

Por último, la labor del contador público, como profesional integral, es fundamental en el desarrollo de la organización desde su área gerencial y financiera, no sólo desde la apropiación de sus conocimientos sino en el libre ejercicio de su criterio dentro de su rol, enmarcado en el deber ser de las personas y de la organización dentro de la sociedad. 


\section{Referencias}

Alexander, D. \& Archer, S. (2005). Guía Miller de Normas Internacionales de Contabilidad/ Normas Internacionales de Información Financiera. Estados Unidos de América: Red Contable Publicaciones.

Ascanio, A. (2005). La estructura económica y financiera de un botel promedio, Contribuciones a la Economía. Fecha de consulta: 10/01/2017. Recuperado de https:// www.eumed.net/eumednet/ce/2005/ aa.htm.

Bohórquez, N. del P. (2014), Implementación de norma internacional de inventarios en Colombia. Innovar Journal. 25, (57). $79-92$.

Cano, A. M. (2010). Análisis de la norma internacional de contabilidad (NIIF/NIC) No. 8 (Políticas contables, cambios en las estimaciones contables y errores) y sus principales efectos en el Estatuto Tributario colombiano. Cuadernos de Contabilidad.11, (28). 123-147.

Congreso de Colombia (2000), Ley 590 por la cual se dictan disposiciones para promover el desarrollo de las micro, pequeñas y medianas empresa.

Congreso de Colombia (2004), Ley 905 por medio de la cual se modifica la Ley 590 de 2000 sobre promoción del desarrollo de la micro, pequeña y mediana empresa colombiana y se dictan otras disposiciones.

Congreso de Colombia (2009), Ley 1314 por la cual se regular los principios y normas de contabilidad e información financiera y de ase- guramiento de información aceptados en Colombia, se señalan las autoridades competentes, el procedimiento para su expedición y se determinan las entidades responsables de vigilar su cumplimiento.

Consejo Técnico de la Contaduría Pública. (2012), Direccionamiento Estratégico del proceso de convergencia de las normas de contabilidad e información financiera y de aseguramiento de la información, con estándares internacionales.

Díaz, M., Gil, J. J. \& Vílchez, P. (2010). Hacia la convergencia mundial del marco conceptual para la preparación de los estados financieros. Contabilidad y negocios, 5 , (9). 19-56.

Espinosa, A. (2015). Tendencias de producción académica alrededor de los retosglobales de la contabilidad en el contexto regional: revisiónde las publicaciones de cinco revistas latinoamericanas. (Trabajo de pregrado). Universidad Militar Nueva Granada. Bogotá, Colombia. Recuperado de https://repository.unimilitar.edu. co/bitstream/handle/10654/7820/

Monografia $\% 20$ tendencias $\% 20$ de $\% 201 \mathrm{a} \% 201$ iteraruta $\% 20$ contable. pdf;jessionid $=63 \mathrm{EDE} 374 \mathrm{~B} 1282 \mathrm{EF}$ 48C029D4206D1BFEF?sequence $=1$

Ferrer, A. M. (2013). Análisis del proceso de convergencia a Normas Internacionales de Contabilidad e Información Financiera desde los factores intrínsecos al sistema contable en Colombia. Cuadernos de Contabilidad. 14, (36). 971 - 1007. 


\section{Referencias}

Fundación IFRS (2010). Material de formación sobre la NIIF para las PYMES (versión 2010-1). United Kingdom.

Hellmann, A. Perera, H. \& Patel, C. (2010), Contextual Issues of the Convergence of International Financial Reporting Standards: The case of Germany, Advances in Accounting, Incorporating Advances in International Accounting. 26, (1). 108-116.

López, C. O. \& Zea, F. (2011), Convergencia a estándares internacionales de información financiera y de aseguramiento en Colombia: análisis de documentos de direccionamiento estratégico de CTCP. Revista Internacional Legis de Contabilidad y Auditoría. (48), 97 - 134.

Marcotrigiano, L. (2013), Reflexiones acerca de la elaboración y presentación de los estados financieros bajo ambiente VEN-NIF PYME, Actualidad contable FACES. 16, (26). 45-81.

Martínez, M. R. (2009), Evaluación financiera y operacional: Aplicada en pymes hoteleras, Revista EAN. (65). 31 - 48.

Martínez, P. C. (2006), El método de estudio de caso: estrategia metodológica de la investigación científica. Pensamiento \& Gestión. (20). 165 - 193.

Mejía, E. (2005), Introducción al pensamiento contable de Richard Mattessich, Revista Internacional Legis de Contabilidad y Auditoría. (24). 135 - 174.
Mejía, E., Montes, C. A. \& Montilla, O. de J. (2008), Fundamentos teóricos del mundo contable común para las PYMES de América Latina: Una alternativa a la regulación contable internacional IASB. Estudios Gerenciales. 24, (107). 59-86.

Menéndez, B. (2010). Optimización de costes en la gestión hotelera. Informe Hoteles \& Resorts. Recuperadode:http:/ / static.hosteltur.com/web/uploads/2010/05/ fdd233c6b0b0b2f3.pdf, Fecha de consulta: 13/12/2016.

Montaner \& Perozo, J. (2008), Formación ética del Contador Público. Caso: Facultad de Ciencias Económicas y Sociales de la Universidad del Zulia, Revista de Ciencias Sociales RCS, 14, (2). 379 - 387.

Nicniif.org (2016). Módulos educativos adicionales sobre la NIIF para las PYMES versión 2015. Fecha de consulta: 20/11/2016. Recuperado de http://www.nicniif. org/home/

Ospina, J. E. \& Villaquirán, C. (2010). La utilidad de la información contable en el proceso de cambio organizacional. Un estudio de caso. Contaduría Universidad de Antioquia. (56). 105-136. Recuperado de https://aprendeenlinea.udea. edu.co/revistas/index.php/cont/article/viewFile/14696/12849

República de Colombia (2013), Decreto 3022 por el cual se reglamenta la Ley 1314 de 2009 sobre el marco técnico normativo de información financiera que conforman el Grupo 2, Colombia. 


\section{Referencias}

República de Colombia (1993), Decreto 2649 por el cual se reglamenta la Contabilidad en General y se expiden los principios o normas de contabilidad generalmente aceptados en Colombia.

Salazar, E. (2013), Efectos de la implementación de la NIIF para las PYMES en una mediana empresa ubicada en la ciudad de Bogotá, Cuadernos de Contabilidad. 14, (35), Colombia, 395-414.
Salazar, C. A., Salazar, E. \& Marín, J. A. (2015), Contabilidad financiera para pequeñas y medianas empresas, Colombia, Bogotá: Legis Editores S.A.

Sánchez, V. \& Holguín, M. (2007), NIIF: Caso práctico de adaptación en una empresa hotelera, Revista de Contabilidad y Dirección, 4. 205 - 229. 